\title{
Avaliação da completude do Sistema de Informação sobre Mortalidade por suicídio na região Sudeste, Brasil, no período de 1996 a 2007
}

\author{
Evaluation of the completeness of the System of Information on \\ Mortality of suicide in the Southeast region, Brazil, from 1996 to 2007 \\ Luciene Bolzam Macente', Eliana Zandonade²
}

\section{RESUMO}

Objetivo: Analisar a completude dos dados do SIM sobre os óbitos por suicídio no ES, Sudeste e Brasil (1996 a 2007), referentes às variáveis demográficas. Métodos: Estudo descritivo analítico baseado em dados secundários. Foram incluídos os óbitos registrados no SIM por suicídio do Espírito Santo, Sudeste e Brasil. Analisaram-se o número absoluto e o percentual de não preenchimento das variáveis nas declarações de óbito (Dos) por suicídio durante o período. Com a finalidade de avaliar os graus de não completude das variáveis sociodemográficas das DOs, adotou-se escore proposto por pesquisadores. As equações do modelo escolhido e as estatísticas de ajuste para os percentuais de dados faltantes ao longo do tempo (valor de $\mathrm{R}^{2}$ e o $\mathrm{p}$-valor do teste $\mathrm{F}$ de adequação do modelo) foram obtidas do programa SPSS. O nível de significância adotado foi de 5\%. Resultados: As equações dos modelos encontrados indicam que as tendências de não completude para algumas variáveis são cres-

\section{Palavras-chave}

Suicídio, declaração de óbito, sistemas de informação. centes, enquanto para outras são decrescentes e estatisticamente significantes. Conclusão: As variáveis sexo e idade apresentaram estabilidade nas taxas no ES, Sudeste e Brasil, com melhoras significativas no preenchimento das DOs desses últimos. Quanto às demais variáveis, o ES destacou-se com os piores índices de preenchimento das DOs por suicídio.

\begin{abstract}
Objective: Analyze the completeness of the data of SIM on the deaths of suicide in ES, Southeast and Brazil (1996 to 2007), referring to the demographic variables. Methods: A descriptive analytical study based on secondary data. The deaths registered in the SIM of suicide in Espirito Santo, Southeast and Brazil were included. The absolute number and percentage of non fulfillment of the variables in the declaration of death of suicide during the period was analyzed. To evaluate the degrees of non completeness of the socio demographic declaration of death variables, a score recommended by the researchers was adopted. The equations of the model chosen and the adjustment statistics for the missing data percentages over time ( $R^{2}$ value and $p$-value of the $F$ test of model adequacy) were obtained from the SPSS program. The level of significance adopted was of 5\%. Results: The equations of the models found show that the tendencies of non complete-
\end{abstract}

1 Universidade Federal do Espírito Santo (UFES), Programa de Pós-Graduação em Saúde Coletiva; Prefeitura de Santa Maria de Jetibá, ES. 2 UFES, Departamento de Estatística e Programa de Pós-Graduação em Saúde Coletiva. 


\section{Keywords}

Suicide, death certificates, information systems. ness for some variables are rising, while for other variables are falling and statistically significant. Conclusion: The sex and age variables indicated stability in the rates in ES, Southeast and Brazil, with significative improvements in the fulfillment of the declarations of death of the latter. As to the other variables, ES turned out to have the worst rates in the fulfillment of the declarations of death of suicide.

\section{INTRODUÇÃO}

O suicídio constitui-se em um grave problema de saúde pública, em especial por seu crescimento entre a população mais jovem?.

Dados da Organização Mundial da Saúde (OMS) mostram que, no ano 2000, aproximadamente 1 milhão de pessoas morreu por suicídio no mundo, o que representa uma morte a cada 40 segundos ${ }^{2-4}$.

Apesar de o Brasil apresentar uma taxa geral de suicídio considerada baixa numa escala mundial (710 lugar quando comparado a outros países), o país ocupa o 90 lugar na lista de países líderes em termos totais de mortes por suicídio 3 . Em certas cidades e regiões, bem como em alguns grupos populacionais (jovens em grandes cidades, indígenas do Centro-Oeste e do Norte e entre lavradores do interior do Rio Grande do Sul), as cifras se aproximam das encontradas em países apontados como de frequência média a elevada ou mesmo as superam ${ }^{5-7}$.

A distribuição dos óbitos por suicídio no país demonstra que os coeficientes variam muito entre as várias regiões (enquanto a região Sudeste concentra 50\% dos registros de suicídio, a região Sul possui os maiores coeficientes de suicídio e a região Norte, os menores índices), não sendo possível afirmar precisamente qual a relação entre esses dados e os problemas relacionados à qualidade do registro das informações. Destaca-se que, no Brasil, assim como no resto do mundo, ainda hoje tanto a disponibilidade quanto a qualidade dos dados referentes aos óbitos são fatores que limitam as pesquisas sobre a mortalidade ${ }^{8,9}$.

Outro fato que merece destaque se refere à ocorrência de subnotificação dos casos de suicídio, os quais podem ser omitidos por vários motivos (religiosos, sociais, culturais, políticos, econômicos etc. ${ }^{10}$. Ainda que não se disponha de uma medida precisa que indique o grau dessa distorção, a qual deve variar no espaço e no tempo, estima-se que ela se encontra entre $20 \%$ e $100 \%$, sempre para menos ${ }^{11}$.

Volpe et al. ${ }^{12}$ afirmam que, apesar de ser difícil mensurar, é provável que os dados referentes à mortalidade por suicídio, em sua maioria, não reflitam a realidade das mortes, por diversos motivos ligados ao próprio fenômeno: a) por ser o suicídio um tema ainda estigmatizante, o que pode induzir a registros de óbitos que não utilizem esse termo; b) por motivos com explicações jurídicas, como o que ocorre em nosso país, onde o suicídio ainda é considerado crime pelo Códi- go Penal, o que resulta em possíveis consequências, como o não atendimento em casos de seguros de saúde, de vida, ou mesmo de possíveis ressarcimentos atribuíveis à vítima ${ }^{13}$; c) relutância do profissional responsável em usar o código para suicídio, o que o leva a registrar o óbito como "morte acidental" ou como "causa indeterminada"; d) a religião, visto que algumas podem ser muito repressoras no que se refere ao suicídio.

Minayo ${ }^{14}$, em revisão sobre o fenômeno do suicídio, assinala o peso dos fatores socioculturais na subnotificação desses casos, mostrando que, no Brasil, por causa da forte influência da cultura judaico-cristã, ocorre de, frequentemente, os familiares tentarem esconder, negociar com quem notifica; num nível mais amplo, a sociedade estigmatiza a família e o sujeito que se suicidou. Além disso, a subnotificação também ocorre por inadequação dos registros, por preenchimento impreciso dos instrumentos de captação de dados, pela existência de cemitérios clandestinos e por destruição de cadáveres.

Considerando que uma base de dados de boa qualidade deve ser completa, fidedigna aos dados originais registrados nas unidades de saúde, sem duplicidades, devendo seus campos ser totalmente preenchidos e consistentes, torna-se necessário avaliar a subnotificação e a confiabilidade dos dados a partir do desenvolvimento de estudos e pesquisas especiais $^{15}$. Nessa perspectiva, a presente pesquisa teve como objetivo analisar a completude dos dados do SIM sobre os óbitos por suicídio no estado do Espírito Santo, na região Sudeste e no Brasil no período de 1996 a 2007, referentes às variáveis demográficas (sexo, faixa etária, raça/cor, escolaridade, estado civil, local de ocorrência).

\section{MÉTODOS}

Todo o processo de pesquisa foi conduzido conforme a Resolução no 196/96 do Conselho Nacional de Saúde (CNS), tendo sido o projeto aprovado pelo Comitê de Ética e Pesquisa da Universidade Federal do Espírito Santo, sob o no 159/09.

Realizou-se estudo descritivo analítico baseado em dados secundários. Foram incluídos os óbitos no período de 1996-2007, ocorridos no estado do Espírito Santo, na região Sudeste brasileira e no Brasil como um todo, registrados no Sistema de Informação sobre Mortalidade (SIM), que 
possuíam como causa básica o suicídio. Esses dados foram obtidos no endereço eletrônico do Departamento de Informática do Sistema Único de Saúde (Datasus) do Ministério da Saúde. Dados sobre estimativas populacionais, que também se encontravam no SIM, foram obtidos do Instituto Brasileiro de Geografia (IBGE) ${ }^{16}$. Os dados foram coletados pelos autores do trabalho no período de março a abril de 2010.

O período selecionado (de 1996 a 2007) para cálculo do número de suicídios refere-se ao período de vigência da Décima Revisão da Classificação Internacional de Doenças (CID10), a qual arrola as categorias $X 60$ a $X 84$.

Foram verificados os itens de algumas variáveis demográficas disponíveis no sistema do Datasus (idade, sexo, estado civil, escolaridade, raça/cor, local de ocorrência), que não foram preenchidas (categoria ignorado). Foi analisado o número absoluto e calculado o percentual de não preenchimento das informações das declarações de óbito (DOs), que são a base de informação do SIM, para as localidades selecionadas (Espírito Santo, região Sudeste e Brasil).

Com a finalidade de avaliar os graus de não completude das variáveis sociodemográficas das DOs, adotou-se, neste estudo, o escore proposto por Romero e Cunha ${ }^{17}$, que, com o objetivo de apresentar uma análise da qualidade dos dados socioeconômicos e demográficos das DOs de menores de um ano, por região e Unidade de Federação (UF), usando dados do SIM (de 1996 a 2001), criaram o seguinte escore: excelente (não completude menor de 5\%), bom (não completude de $5 \%$ a $10 \%$ ), regular (não completude de $10 \%$ a $20 \%$ ), ruim (não completude de $20 \%$ a $50 \%$ ) e muito ruim (não completude de $50 \%$ ou mais).
De posse dos dados, foram elaborados os gráficos para as variáveis estudadas conforme cada local de estudo (Espírito Santo, Sudeste brasileiro e Brasil) ao longo do tempo. A análise do melhor formato do gráfico e o valor do coeficiente de ajuste $R^{2}$ indicaram a melhor curva a ser ajustada aos dados (linear, quadrático, exponencial, logaritmo, modelo inverso etc.). As equações do modelo escolhido (o melhor para cada caso) e as estatísticas de ajuste para os percentuais de dados faltantes ao longo do tempo (valor de $\mathrm{R}^{2}$ e o $\mathrm{p}$-valor do teste $\mathrm{F}$ de adequação do modelo) foram obtidas do programa SPSS, versão 15.0. O nível de significância adotado foi de 5\%.

\section{RESULTADOS}

No período de estudo (de 1996 a 2007), ocorreram 1.553 óbitos por suicídio no estado do Espírito Santo, 35.262 óbitos na região Sudeste e 91.364 óbitos por essa causa em todo o Brasil.

A incidência de suicídios nesse período variou de 3,1 (ano de 1999) a 4,9 (ano de 2005)/100.000 habitantes no Espírito Santo; de 3,5 (ano de 2000) a 4,3 (ano de 1997)/100.000 habitantes na região Sudeste e de 4,0 (ano de 1999) a 4,7 (ano de 2007)/100.000 no Brasil.

Foram calculadas as porcentagens de não preenchimento de algumas variáveis demográficas (idade, sexo, estado civil, escolaridade, raça/cor e local de ocorrência) das declarações de óbito (DOs) relativas aos casos de suicídio para o estado do Espírito Santo, no período de estudo (de 1996 a 2007), conforme mostra a tabela 1. Apresentam-se, ainda, os resultados do escore proposto por Romero e Cunha ${ }^{17}$.

Tabela 1. Avaliação da não completude das DOs por suicídio no estado do Espírito Santo, segundo idade, sexo, estado civil, escolaridade, raça/cor e local de ocorrência, no período de 1996 a 2007

\begin{tabular}{|c|c|c|c|c|c|c|c|c|c|c|c|c|c|}
\hline \multirow[b]{2}{*}{ Ano } & \multicolumn{2}{|c|}{ Idade } & \multicolumn{2}{|c|}{ Sexo } & \multicolumn{2}{|c|}{ Estado civil } & \multicolumn{2}{|c|}{ Escolaridade } & \multicolumn{2}{|c|}{ Raça/cor } & \multicolumn{2}{|c|}{ Local de ocorrência } & \multirow{2}{*}{$\begin{array}{l}\text { Total de } \\
\text { óbitos/ } \\
\text { suicídio }\end{array}$} \\
\hline & $\%$ & ESCORE & $\%$ & ESCORE & $\%$ & ESCORE & $\%$ & ESCORE & $\%$ & ESCORE & $\%$ & ESCORE & \\
\hline 1996 & 0 & E & 0 & $E$ & 6,2 & B & 85,7 & MR & 100 & MR & 0,9 & $E$ & 112 \\
\hline 1997 & 0 & $E$ & 0,8 & $\mathrm{E}$ & 0,8 & $\mathrm{E}$ & 81,9 & MR & 66,4 & MR & 3,4 & E & 116 \\
\hline 1998 & 0 & $E$ & 0,8 & $\mathrm{E}$ & 4 & $E$ & 84,7 & MR & 54,8 & MR & 0 & $E$ & 124 \\
\hline 1999 & 0 & $E$ & 0 & $\mathrm{E}$ & 3,3 & $E$ & 65,9 & MR & 29,7 & $\mathbf{R}$ & 6,6 & B & 91 \\
\hline 2000 & 0 & $E$ & 0 & $\mathrm{E}$ & 1,9 & $\mathrm{E}$ & 65,0 & MR & 24,5 & $\mathbf{R}$ & 4,7 & E & 106 \\
\hline 2001 & 0 & E & 0 & $\mathrm{E}$ & 5,2 & B & 65,5 & MR & 23,3 & $\mathbf{R}$ & 1,7 & E & 116 \\
\hline 2002 & 0 & $\mathrm{E}$ & 0 & $\mathrm{E}$ & 5,4 & B & 71,1 & MR & 23,4 & $\mathbf{R}$ & 3,9 & $\mathrm{E}$ & 128 \\
\hline 2003 & 1,3 & $\mathrm{E}$ & 0 & $\mathrm{E}$ & 8,6 & B & 78,6 & MR & 20,6 & $\mathbf{R}$ & 5,3 & B & 150 \\
\hline 2004 & 0 & E & 0 & $\mathrm{E}$ & 9,3 & B & 76,8 & MR & 21,8 & $\mathbf{R}$ & 4,6 & E & 151 \\
\hline 2005 & 0,6 & $\mathrm{E}$ & 0 & $\mathrm{E}$ & 15,6 & $\mathrm{R}$ & 74,7 & MR & 18,7 & RE & 7,2 & B & 166 \\
\hline 2006 & 0 & E & 0 & $\mathrm{E}$ & 11,4 & RE & 79,6 & MR & 26,7 & $\mathbf{R}$ & 8,9 & B & 157 \\
\hline 2007 & 0 & E & 0 & $\mathrm{E}$ & 14,7 & RE & 83,8 & MR & 19,1 & RE & 2,9 & E & 136 \\
\hline
\end{tabular}

E: excelente (menor de $5 \%$ ); B: bom (5\% a 10\%); RE: regular (10\% a 20\%); R: ruim (20\% a 50\%) e MR: muito ruim (50\% ou mais) $)^{17}$. 
Foram calculados modelos de regressão linear simples para os dados da porcentagem de não completude segundo a variável ano $(X)$ para o Espírito Santo, para o Sudeste e para o Brasil. As equações dos modelos encontrados, valor de $R^{2}$ e respectivos $p$-valores do teste $F$ indicam, como mostra a tabela 2 , que as tendências para algumas variáveis são crescentes, enquanto para outras são decrescentes e estatisticamente significantes. Para os resultados estatisticamente significantes, calculou-se o percentual (\%) de crescimento.

Com relação à idade, verificou-se que, no período em análise, não foram observados problemas referentes ao preenchimento das DOs por suicídio no estado do Espírito Santo. Mesmo nos anos de 2003 e 2005, em que a porcentagem de não preenchimento das DOs foi de 1,3\% e 0,6\%, respectivamente, observa-se que, conforme o escore proposto por Romero e Cunha ${ }^{17}$, por serem esses valores menores de $5 \%$, eles correspondem a um nível excelente de preenchimento.

Em relação ao estudo de tendência, observou-se (para a variável idade) tendência decrescente dos percentuais de não completude das DOs referentes aos óbitos por suicídio, apenas para o Brasil, de $-73,5 \%(p=0,002)$, enquanto os percentuais para o estado do Espírito Santo e da região
Sudeste mantiveram-se estáveis. Verifica-se, ainda, que, enquanto para o Espírito Santo ocorre estabilidade referente a não completude da variável idade durante os anos de 1996 a 2002, ocorreram oscilações após esse período (2003 a 2005), as quais se revelam as mais altas entre os locais estudados. Para a região Sudeste e para o Brasil, o não preenchimento da informação idade nas DOs por suicídio manteve índices elevados ao longo do tempo, chamando a atenção, contudo, o fato de que, em termos de Brasil, observa-se melhora gradativa das informações.

Quanto ao sexo, observa-se que 0,8\% das DOs por suicídio no estado do Espírito Santo não foi preenchido tanto no ano de 1997 quanto no ano de 1998, apresentando melhora na qualidade das informações de 1999 a 2007. Verificou-se, no período estudado, uma tendência decrescente dos percentuais de não completude das DOs por suicídio, destacando-se que ela foi de $-99,1 \%(p=0,011)$ para a região Sudeste e de -80,0\% ( $p=0,034)$ para o Brasil, mantendo-se estável para o Espírito Santo. Observa-se, ainda, a ocorrência de certa constância na melhora da qualidade do preenchimento das DOs tanto na região Sudeste quanto no Brasil, ao longo do período, enquanto o Espírito Santo apresenta os maiores

Tabela 2. Resultado da análise de tendências e modelo ajustado do não preenchimento das DOs para as variáveis sexo, idade, estado civil, escolaridade, raça/cor e local de ocorrência, referentes ao estado do Espírito Santo, à região Sudeste e ao Brasil, no período de 1996 a 2007

\begin{tabular}{|c|c|c|c|c|c|c|}
\hline Variável & Local & Modelo & $R^{2}$ & p-valor & Tendência & Crescimento \% \\
\hline \multirow[t]{3}{*}{ Sexo } & ES & Constante & 0,270 & 0,083 & - & - \\
\hline & Sudeste & Linear & 0,494 & 0,011 & Decrescente & $-99,1$ \\
\hline & Brasil & Linear & 0,376 & 0,034 & Decrescente & $-80,0$ \\
\hline \multirow[t]{3}{*}{ Idade } & ES & Constante & 0,065 & 0,426 & - & - \\
\hline & Sudeste & Constante & 0,255 & 0,094 & - & - \\
\hline & Brasil & Linear & 0,644 & 0,002 & Decrescente & $-73,5$ \\
\hline \multirow[t]{3}{*}{ Estado civil } & ES & Linear & 0,706 & 0,001 & Crescente & 57,5 \\
\hline & Sudeste & Linear & 0,688 & 0,001 & Crescente & 50,7 \\
\hline & Brasil & Linear & 0,487 & 0,012 & Crescente & 13,0 \\
\hline \multirow[t]{3}{*}{ Escolaridade } & ES & Constante & 0,000 & 0,958 & - & - \\
\hline & Sudeste & Logaritmo & 0,803 & 0,001 & Decrescente & $-50,2$ \\
\hline & Brasil & Logaritmo & 0,838 & 0,001 & Decrescente & $-54,8$ \\
\hline \multirow[t]{3}{*}{ Raça/cor } & ES & Inverso & 0,947 & 0,001 & Decrescente & $-80,8$ \\
\hline & Sudeste & Inverso & 0,971 & 0,010 & Decrescente & $-96,1$ \\
\hline & Brasil & Inverso & 0,985 & 0,006 & Decrescente & $-95,4$ \\
\hline \multirow[t]{3}{*}{ Local de ocorrência } & ES & Constante & 0,313 & 0,058 & - & - \\
\hline & Sudeste & Constante & 0,271 & 0,083 & - & - \\
\hline & Brasil & Constante & 0,093 & 0,336 & - & - \\
\hline
\end{tabular}

Linear: incidência $=a+b^{*}$ ano 
índices de não preenchimento dessa informação durante os primeiros anos em questão, com posterior estabilização.

No que se refere ao estado civil, os resultados demonstram problemas com o preenchimento dessa variável nas DOs por suicídio no Espírito Santo, pois sua porcentagem de não preenchimento variou de 0,8\%, em 1997, a 14,7\%, em 2007, apontando uma tendência ascendente das porcentagens de não preenchimento dessa informação a partir de 2005. Para os resultados de tendência, verificou-se tendência crescente dos percentuais de não completude das DOs no Espírito Santo, na região Sudeste e no Brasil, com valores de $57,5 \%(p=0,001), 50,7 \%(p=0,001)$ e $13,0 \%(0,012)$ de crescimento, respectivamente, como mostra a figura 1 .

Com relação à escolaridade, verifica-se que o não preenchimento das informações referentes a essa variável no Es- pírito Santo variou de 85,7\%, em 2000, a 83,8\%, em 2007. Observa-se que não houve melhora na qualidade do preenchimento dessa informação, mantendo-se altos índices de não preenchimento durante os 12 anos em análise. Observou-se tendência decrescente dos percentuais de não completude das DOs na região Sudeste, com -50,2\% ( $p=0,001)$, e no Brasil, com -54,8\% ( $p=0,001)$, mantendo-se estável para o estado do Espírito Santo. A figura 2 destaca tais resultados.

Para a variável raça/cor, o percentual de não preenchimento variou de 100\%, em 1996, a 19,1\%, em 2007, no Espírito Santo. Constata-se que houve melhora na qualidade do preenchimento do dado nas DOs, sendo possível observar uma curva descendente em termos de não preenchimento das informações. Contudo, chama a atenção o fato de o não preenchimento dessa variável continuar alto no Estado.

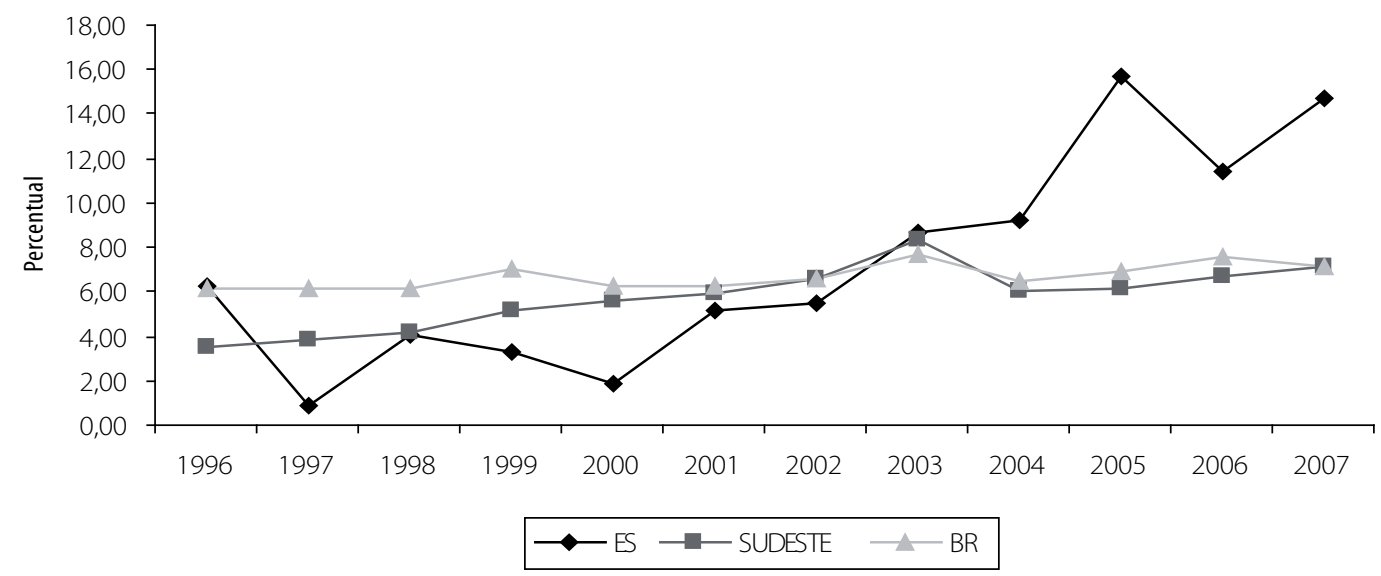

Figura 1. Percentual de não preenchimento das DOs referentes aos casos de suicídio segundo estado civil, para o estado do Espírito Santo, a região Sudeste e o Brasil, no período de 1996 a 2007.

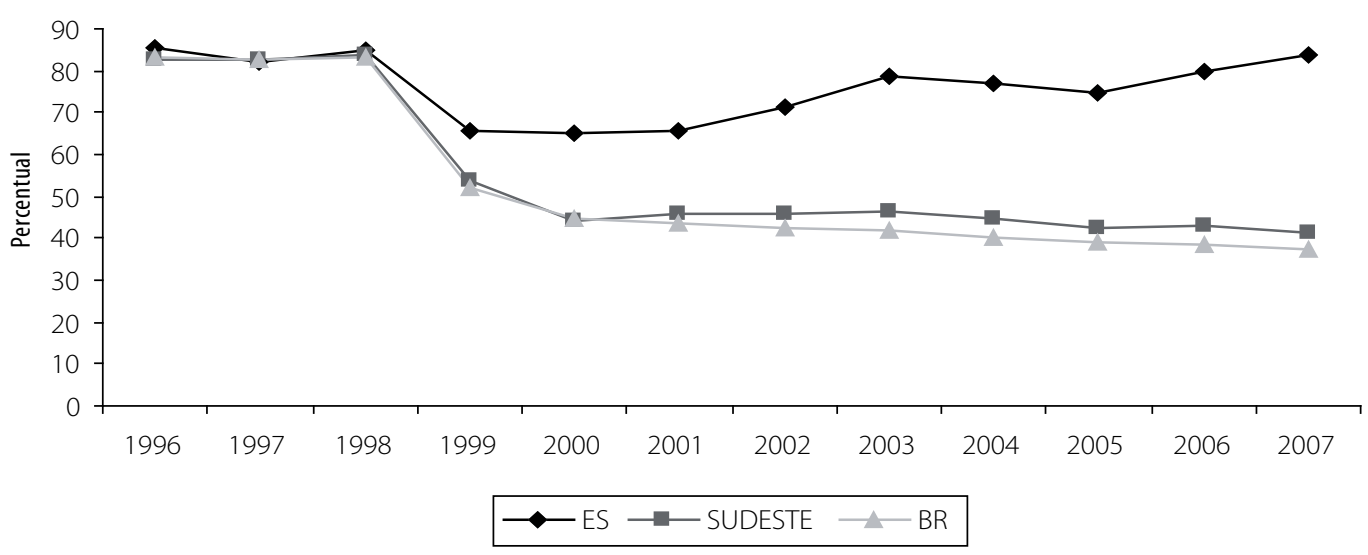

Figura 2. Percentual de não preenchimento das DOs referentes aos casos de suicídio segundo escolaridade, para o estado do Espírito Santo, a região Sudeste e o Brasil, no período de 1996 a 2007. 
Ainda em relação à variável raça/cor, verificou-se tendência decrescente dos percentuais de não preenchimento das DOs no Espírito Santo, na região Sudeste e no Brasil, com $-80,8 \%(p=0,001),-96,1 \%(p=0,010)$ e $-95,4(p=0,006)$ respectivamente. A figura 3 evidencia tais resultados, ao ilustrar que, de modo geral, houve melhora na completude das informações referentes à variável raça/cor nas três regiões. Contudo, essa melhora foi menor no estado do Espírito San- to, o qual se manteve com os maiores índices de não completude no período.

Quanto ao local de ocorrência dos óbitos, foi possível observar uma variação, no período estudado, de 0,9\%, em 1996, a 2,9\%, em 2007, com oscilações nos dados do Espírito Santo. Verifica-se que os percentuais de não completude das DOs, tanto no Espírito Santo quanto na região Sudeste e no Brasil, mantiveram-se estáveis no período estudado, como demonstra a figura 4.

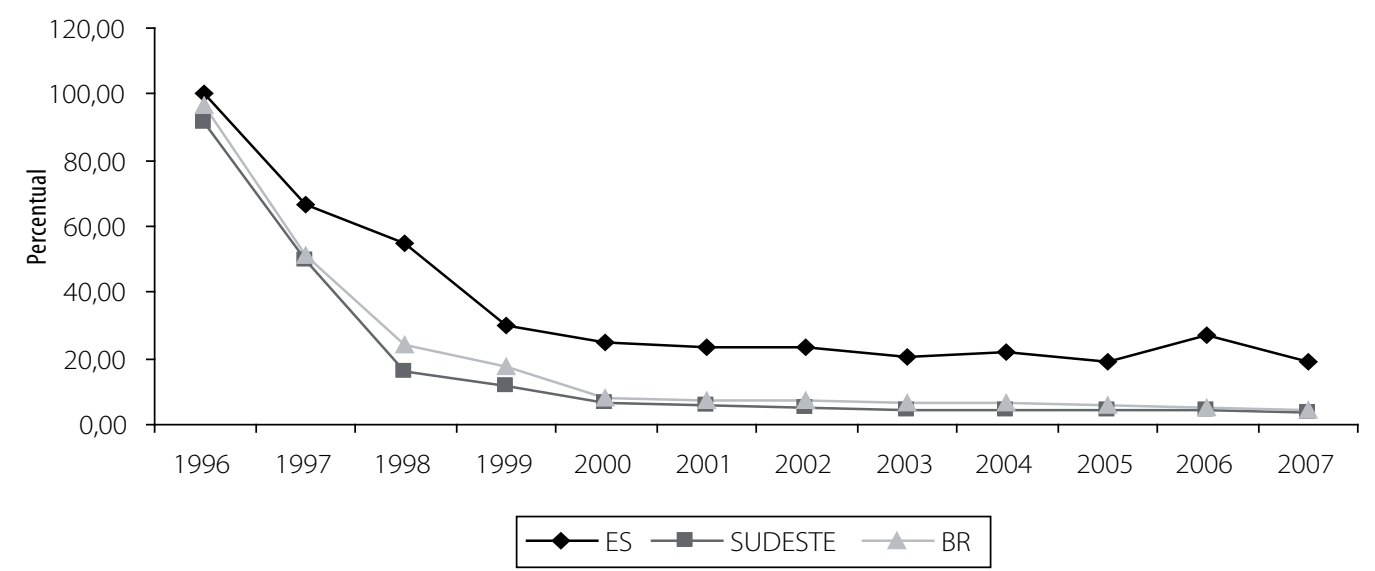

Figura 3. Percentual de não preenchimento das DOs referentes aos casos de suicídio segundo raça/cor, para o estado do Espírito Santo, a região Sudeste e o Brasil, no período de 1996 a 2007.

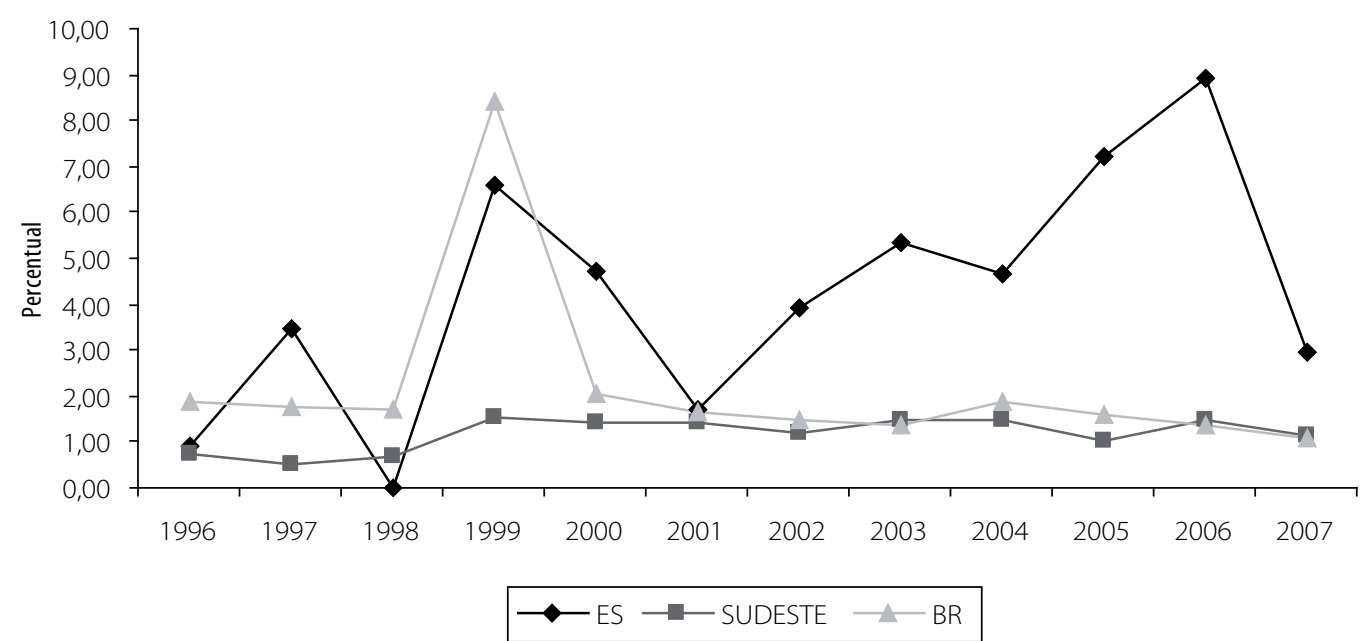

Figura 4. Percentual de não preenchimento das DOs referentes aos casos de suicídio segundo local de ocorrência do óbito, para o estado do Espírito Santo, a região Sudeste e o Brasil, no período de 1996 a 2007.

\section{DISCUSSÃO}

A epidemiologia tem muito a contribuir no processo de enfrentamento desse grave problema que é o suicídio, pois responder a questões como estas: Como é o padrão do comportamento suicida? Qual a faixa etária mais acometida?
Em relação ao sexo, quem se suicida mais, os homens ou as mulheres? Qual a tendência de crescimento das taxas em cada grupo? e muitas outras é fundamental para o planejamento de políticas de saúde pública ${ }^{12}$. Com isso, os sistemas de informação assumem um papel fundamental enquanto fontes de dados. Diminuir as subnotificações dos casos de 
suicídio, assim como primar pela qualidade no preenchimento dos documentos que fazem parte de sua base, é um fator imprescindível para a compreensão e a prevenção do fenômeno.

Segundo o Ministério da Saúde, apesar da subnotificação, observa-se um aumento dos casos de suicídio no país, chegando a um acréscimo de $34 \%$ em suas taxas nos anos de 1979 a 1997. No ano de 1997, 6.920 pessoas cometeram suicídio ${ }^{18}$. Em 2003, foram 7.861 casos, enquanto, no ano de 2004, o SIM captou 8.017 óbitos por suicídio, o que representou $0,8 \%$ do total de óbitos no país ${ }^{19}$. Na presente pesquisa, também é possível verificar o número expressivo desses óbitos no país (91.364 casos, nos últimos 12 anos), principalmente por se tratar de mortes evitáveis.

No que se refere à qualidade dos dados do SIM, várias pesquisas vêm mostrando que, para todos os tipos de óbito, o preenchimento dos atestados de óbito não é satisfatório. Destacam as pesquisas que o atestado é um formulário que deve ter todos os itens preenchidos, e o fato de isso não acontecer leva à conclusão de que não se presta a devida atenção nem se compreende a importância do atestado como fonte de informações ${ }^{20}$.

Segundo o Ministério da Saúde ${ }^{21}$ (2009), a frequência de informação "ignorada" ou, simplesmente, "não preenchida" para as variáveis sexo e idade vem se mantendo, no tempo, sempre em níveis bastante baixos, permitindo que essas variáveis possam ser bem analisadas do ponto de vista epidemiológico. Afirma o Ministério não haver dúvida de que o SIM vem melhorando, acentuadamente, quer quanto à cobertura, quer quanto à qualidade de seus dados, ainda que, quanto a esse último aspecto, a informação sobre a causa básica de morte possa deixar um pouco a desejar. Entretanto, pode-se afirmar que mesmo essa variável apresentou, nas duas últimas décadas, indícios de sensível melhora. Nesse sentido, destaca-se que os resultados encontrados na presente pesquisa se encontram em conformidade com as observações do Ministério da Saúde, pois enquanto, para a variável sexo, foi constatada tendência decrescente das taxas de não completude das DOs por suicídio, na região Sudeste e no Brasil, o Espírito Santo apresentou taxas de não completude estáveis no período. Para a variável idade, verifica-se tendência decrescente das taxas de não completude das DOs por suicídio apenas para o Brasil, enquanto as taxas para o estado do Espírito Santo e para a região Sudeste se mantiveram estáveis.

Romero e Cunha ${ }^{17}$ observaram que, no período de 1996 a 2001, ocorreu um alto grau de omissão de determinadas variáveis das DOs em todas as regiões brasileiras. Também os resultados encontrados na presente pesquisa apontam o alto grau de omissão de determinadas variáveis nas DOs por suicídio nas três regiões analisadas (Espírito Santo, região Sudeste e Brasil).

Romero e Cunha ${ }^{17}$ destacam ainda que, ao considerarem a proporção de informação ignorada nas DOs, criaram um escore a partir da não completude de suas variáveis sociodemográficas e observaram que, a partir do ano de 1998, essas informações passaram a ser cada vez menos preenchidas, chegando, em 2001, a apresentar resultado muito ruim para todas as regiões do país. Ressaltam os autores que as variáveis escolaridade materna e raça/cor do recém-nascido foram as únicas que apresentaram melhorias acentuadas no período. Contudo, apesar de a primeira ter melhorado expressivamente, continua com completude ruim nas regiões Norte, Sul e Centro-oeste (entre $20 \%$ e $50 \%$ ), enquanto em relação à segunda verifica-se melhor completude para o ano de 2001 nas regiões Norte e Sul, embora a omissão dos dados ainda seja maior que $10 \%$. Já no que se refere às demais regiões, destacam que a completude da variável raça/cor é ruim ou muito ruim ${ }^{17}$.

Na presente pesquisa, ao utilizar o escore proposto pelos autores para avaliar a questão da não completude das variáveis sociodemográficas das DOs por suicídio no estado do Espírito Santo, também se verificou que os piores resultados diziam respeito às variáveis escolaridade e raça/cor, chamando a atenção ainda a relativa piora das informações relativas à variável estado civil a partir do ano de 2006.

Com o objetivo de estudar a qualidade do preenchimento da DO, segundo as variáveis raça/cor, estado civil e escolaridade geral, o Ministério da Saúde ${ }^{19}$ destaca ainda os seguintes resultados para o ano de 2003:

a) Raça/cor - Variável introduzida na DO no ano de 1996, atendendo a uma reivindicação da Liga Nacional para a Valorização da Raça Negra. Em seus primeiros anos de vigência, o preenchimento foi bem baixo, mas, com o passar do tempo, foi se aprimorando. No ano de 2003, para o Brasil, o índice de ignorado/branco foi de 10,3\%, enquanto, em termos regionais, o Nordeste teve os valores mais altos (21,3\%), e as demais apresentaram percentuais abaixo de $10 \%$. No ano em que foi incluído o quesito raça/cor na DO (1996), o percentual de não informados foi de 96,7\%. Em 1997, apesar da melhoria no preenchimento, o percentual de não informados ainda foi superior a 50\%. A partir de 2000, o preenchimento torna-se mais robusto, chegando a $15 \%$ os não informados. Na presente pesquisa, também foi possível verificar tendência decrescente das taxas de não preenchimento da variável raça/cor nas DOs por suicídio no Espírito Santo, na região Sudeste e no Brasil. Isso mostra melhora na completude das informações referentes à variável raça/cor nas três regiões, apesar de essa melhora ter sido menor no estado do Espírito Santo, o qual se manteve com os maiores índices de incompletude no período.

b) Estado civil - Variável modificada a partir de 1998, desde então tem apresentado um padrão de preenchimento um tanto baixo na maioria das UFs, o que 
foi observado em 2003. Nesse ano, apenas 11 UFs apresentaram valores na casa de um dígito, enquanto as demais tiveram índices entre 11,8\% (Espírito Santo) e 34,8\% (Sergipe). Tais dados se encontram em conformidade com os resultados encontrados na presente pesquisa, onde foi possível verificar tendência crescente das taxas de não completude dessa variável nas DOs por suicídio no Espírito Santo, na região Sudeste e no Brasil, destacando-se uma linha ascendente de não completude da variável ao longo do tempo, em especial no Espírito Santo, o qual demonstra acentuada piora na qualidade da informação a partir de 2003.

c) Escolaridade geral - Esta variável também teve suas alternativas modificadas a partir de 1998. No ano de 2003, apresentou os percentuais de ignorado/em branco muito altos, na casa de dois dígitos, para todas as áreas geográficas (Brasil, regiões e estados). Os resultados encontrados na presente pesquisa diferem um pouco desses dados, visto ter sido possível observar uma tendência decrescente das taxas de não completude da variável escolaridade nas DOs por suicídio na região Sudeste e no Brasil, mantendo-se estável para o estado do Espírito Santo. Nesse sentido, evidenciou-se melhora no preenchimento da informação nas DOs por suicídio na região Sudeste e no Brasil, com exceção do Espírito Santo, o qual manteve o índice de não completude da informação.

Conforme esses dados, as proporções das variáveis raça/ cor, estado civil e escolaridade, com preenchimento ignorado/branco na declaração de óbito no ano de 2003, para as regiões selecionadas no presente estudo, foram: a) raça/cor: 27,6 para o Espírito Santo, 7,4 para a região Sudeste e 10,3 para o Brasil; b) estado civil: 11,8 para o Espírito Santo, 7,3 para a região Sudeste e 10,4 para o Brasil; e c) escolaridade: 58,7 para o Espírito Santo, 39,8 para a região Sudeste e 39,6 para o Brasil ${ }^{19}$. Novamente se evidencia que o Espírito Santo, ao ser comparado com a região Sudeste e com o Brasil, apresentou os piores índices de não completude para essas variáveis, fato também evidenciado no presente estudo.

No que se refere à variável local de ocorrência do óbito, $\operatorname{Costa}^{22}$ (2008), com o objetivo de avaliar a completude no preenchimento das variáveis da declaração de óbito de menores de 1 ano residentes em Pernambuco no período de 1997 a 2005, encontrou, no que se refere às variáveis relacionadas ao momento do óbito, um decréscimo no número de municípios com preenchimento excelente, exceto para variável local de ocorrência, que apresentou um incremento, chegando a 2005 com todos os municípios atingindo esse escore (excelente). Tais resultados não foram observados na presente pesquisa, visto que, aí, se evidenciou o contrário, ou seja, as taxas de não completude da variável local de ocorrência das DOs por suicídio tanto no Espírito Santo quanto na região Sudeste e no Brasil como um todo mantiveramse estáveis no período estudado. Destacam-se oscilações ao longo do tempo de estudo, principalmente no Espírito Santo, que apresenta os maiores índices de não completude da variável.

Muitos autores atribuem essa falta de preenchimento das variáveis das DOs a fatores como a pouca importância dada à questão nos currículos das escolas médicas, a deficiência no ensino para o preenchimento dos documentos dos registros vitais, assim como a pouca valorização das DOs como documento de essencial importância epidemiológi$\mathrm{ca}^{20,23,24}$. Apontam, ainda, como hipótese, a possibilidade de a maioria dos médicos pensar que o atestado é apenas uma formalidade que serve para o sepultamento. Com isso, esses profissionais ignoram, na maioria das vezes, o fato de esse material ser importante para a saúde pública e para estudos epidemiológicos ${ }^{20}$.

\section{CONCLUSÃO}

Verificou-se uma incidência de suicídio sem grandes diferenças entre as regiões estudadas. Quanto à não completude das variáveis demográficas, destaca-se que, de modo geral, as variáveis sexo e idade apresentaram estabilidade tanto no Espírito Santo quanto na região Sudeste e no Brasil, com melhoras significativas no preenchimento das DOs destes últimos. Já no que se refere às demais variáveis (estado civil, escolaridade, raça/cor e local de ocorrência), o Espírito Santo destacou-se como aquele que apresentou os piores índices de preenchimento das DOs por suicídio. Tais resultados estão de acordo com os dados de outras pesquisas, o que enfatiza a necessidade de se estabelecerem estratégias de enfrentamento para a questão, em especial no estado do Espírito Santo.

\section{REFERÊNCIAS}

1. Feijó M. Suicídio entre a razão e a loucura. São Paulo: Lemos Editorial; 1998.

2. WHO (World Health Organization). Figures and facts about suicide. Geneva; 1999.

3. Organização Mundial da Saúde (OMS). Relatório sobre a saúde no mundo 2001: saúde mental - nova concepção, nova esperança. Genebra; 2001.

4. Bahls Saint-Clair, Botega NJ. Epidemiologia das tentativas de suicídio e dos suicídios. In: Mello MF, Mello AAF, Kohn R. Epidemiologia da saúde mental no Brasil. Porto Alegre: Artmed; 2007, p. 151-71.

5. WHO (World Health Organization). The World Health Report 2003: shaping the future. Geneve: World Health Organization; 2003.

6. Botega NJ. Suicídio: saindo da sombra em direção a um Plano Nacional de Prevenção. Rev Bras Psiquiatr. 2007;29(1):7-8.

7. Lovisi GM, Santos AS, Legay L, Abelha L, Valencia E. Análise epidemiológica do suicídio no Brasil entre 1980 e 2006. Rev Bras Psiquiatr. 2009;31(Supl II):S86-93. 
8. Werlang BSG, Botega NJ. Comportamento suicida. Porto Alegre: Artmed; 2004.

9. Vasconcelos AMN. A qualidade das estatísticas de óbito no Brasil. Rev Bras Estudos Pop. 1998;15(1):115-24.

10. Brasil. Ministério da Saúde. Impacto da violência na saúde dos brasileiros. Brasília: Ministério da Saúde; 2005.

11. Bertolote JM, Fleischmann A. Suicídio e doença mental: uma perspectiva global. In: Werlang BG, Botega NJ. Comportamento suicida. Porto Alegre: Artmed; 2004, p. 35-44.

12. Volpe FM, Corrêa H, Barrero SP. Epidemiologia do suicídio. In: Corrêa H, Barrero SP. Suicídio: uma morte evitável. São Paulo: Atheneu; 2006, p. 10-27.

13. Dias ML. Suicídio: testemunhos de adeus. São Paulo: Brasiliense; 1997.

14. Minayo MCS. A autoviolência, objeto da sociologia e problema de saúde pública. Cad Saude Publica. 1998;14(2):421-8

15. Brasil. Ministério da Saúde. Roteiro para uso do SINAN net, análise da qualidade da base de dados e cálculo de indicadores epidemiológicos e operacionais (Versão Preliminar) Brasília - DF; 2008

16. Brasil. Ministério da Saúde. Departamento de Informática do SUS. 2010 Abril [acessado 2010 abr. 7]. Disponível em: http://www.datasus.gov.br.

17. Romero DE, Cunha CB. Avaliação da qualidade das variáveis socioeconômicas e demográficas dos óbitos de crianças menores de um ano registrados no Sistema de In- formações sobre Mortalidade do Brasil (1996/2001). Cad Saude Publica, Rio de Janeiro. 2006;22(3):673-84

18. Brasil. Ministério da Saúde. Política Nacional de Redução da Morbimortalidade por Acidentes e Violência. Secretaria de Políticas de Saúde. Informes Técnicos Institucionais. Rev Saude Publica. 2000;34(4):427-30

19. Brasil. Ministério da Saúde. Saúde Brasil 2005: uma análise da situação de saúde no Brasil; 2005.

20. Fonseca LAM, Laurenti R. A qualidade da certificação médica da causa de morte em São Paulo. Rev Saude Publica. 1974;9:21-9.

21. Brasil. Ministério da Saúde. A experiência brasileira em sistemas de informação em saúde. Brasília: Editora do Ministério da Saúde; 2009.

22. Costa JMBS. Avaliação da completude das variáveis da declaração de óbitos de menores de um ano residentes em Pernambuco, Brasil, 1997-2005. Ciên Saúde Coletiva. 2008 [acesso em 2010 abr. 7]. Disponível em: http://www.abrasco.org.br/cienciaesaudecoletiva/artigos/artigo_int.php?id_artigo $=4043$.

23. Laurenti R. Fatores de erros na mensuração da mortalidade infantil. Rev Saude Publica. 1975;10:237-42.

24. Stuque $\mathrm{CO}$, Cordeiro JA, Cury PM. Avaliação dos erros ou falhas de preenchimento dos atestados de óbito feitos pelos clínicos e pelos patologistas. J Bras Patol Med Lab. 2003:39:361-4. 